\title{
Editorial
}

\section{Patient Blood Management in Cardiac Surgery and ECMO: The Indian Scenario in 2021}

\author{
Poonam Malhotra Kapoor ${ }^{1}$ \\ ${ }^{1}$ Department of Cardiac Anaesthesia and Critical Care, All India \\ Institute of Medical Sciences, New Delhi, India \\ J Card Crit Care 2021;5:181-183.
}

Cardiac surgery and extracorporeal membrane oxygenation (ECMO) both are thrombogenic procedures, wherein coagulopathy ensues. However, patients who are bleeding need not to be coagulopathic. Hemodilution, hypothermia, and acidosis all worsen coagulopathy. If this process continues, without correction, a consumptive coagulopathy with a dilution coagulopathy (disseminated intravascular coagulation [DIC]) is formed, with immense morbidity and mortality in the later stages, mandating resuscitation and immense morbidity and mortality. Early detection of risk to bleeding and thrombosis prevents inadvertent side effects of anticoagulation. This entails detailed patient blood management (PBM) protocols that are in most centers lacking, Is it feasible in 2021? ${ }^{1}$

There is thus an urgent need of framing protocols and guidelines to perform appropriate tests to judiciously select patients who may be undergoing a cardiac surgery/ECMO, so that the patient is not at a risk of going into a DIC! Also, the need for restrictive blood component transfusions, in these precarious patients, is an urgent measure needed today. As per the recent European Association of Cardiothoracic Anaesthesiology (EACTA) and Indian PBM guidelines, this needs a diligent multimodal, multidisciplinary approach today. $^{2,3}$

\section{No Coagulopathy, No Blood: Is It Feasible Today?}

In the literature, data on anticoagulation practices (in cardiac patient and also both types of ECMO patients-the venovenous [VV] and venoarterial [VA] ECMO), the coagulation profile, and their ability to generate thrombin (the principal clotting factor that maintains the balance between bleeding and thrombosis) is scarce. Addition of an anticoagulant like heparin, argatroban, bivalirudin, or fibrin threads leads to an increase in thrombin time. Activation of thrombin leads to cleavage of fibrinogen into fibrin monomers. ${ }^{1}$ The fibrin threads crosslink to form with the adhering platelets a stable clot. So, an increase in thrombin suggests decreased fibrinogen levels in the body leading to conditions like DIC. ${ }^{2-4}$

\section{Role of Point of Care Testing in Monitoring Hemostasis}

To gain more knowledge into the process of hemostasis in these patients, cardiac critical care, the application of point of care viscoelastic test is very useful (- Figs. 1-5). ${ }^{5}$ Whether we apply Sonoclot/thromboelastography (TEG)/rotational thromboelastometry (ROTEM) or platelet mapping/platelet aggregometry analysis to the mechanics of blood thromboelastometry measures targeting the platelet function is much better a test to do for quick and prompt decision making rather than doing conventional laboratory tests. Conventional tests like the activated coagulation time (ACT), international normalized ratio, activated partial thromboplastin time, antithrombin III levels, platelet count, and antithrombin test are all plasma-based tests. None of these are platelet-centered tests, the platelet function tests as we see on the Sonoclot/ Rotational Thromboelastometry (ROTEM)/ Thromboelastography (TEG) as shown in - Figs. 1 to 5 . The PFson shows platelet dysfunction within 20 minutes on the Sonoclot machine (-Fig. 5) and the A6 or decreased maximum slope(MS) shows deranged platelet function (thrombocytopenia on ECMO-suggesting high heparin dose and hyperfibrinolysis, in the ECMO patientscorrelates with decreased FIBTEM [Fibrinogen Thromboelastometry]) (-Fig. 3). ${ }^{6}$

\footnotetext{
Address for correspondence Poonam Malhotra Kapoor, MD, DNB, MNAMS, FISCU (Hony), FIACTA (Hony), FTEE (Hony), Department of Cardiac Anaesthesia and Critical Care, All India Institute of Medical Sciences, New Delhi 110048, India (e-mail: drpooonamaiims@gmail. com).
}

DOI https://doi.org/ $10.1055 / \mathrm{s}-0042-1742451$. ISSN 2457-0206.

\footnotetext{
(c) 2022. Official Publication of The Simulation Society (TSS), accredited by International Society of Cardiovascular Ultrasound (ISCU). All rights reserved.

This is an open access article published by Thieme under the terms of the Creative Commons Attribution-NonDerivative-NonCommercial-License, permitting copying and reproduction so long as the original work is given appropriate credit. Contents may not be used for commercial purposes, or adapted, remixed, transformed or built upon. (https://creativecommons.org/ licenses/by-nc-nd/4.0/)

Thieme Medical and Scientific Publishers Pvt. Ltd., A-12, 2nd Floor, Sector 2, Noida-201301 UP, India
} 

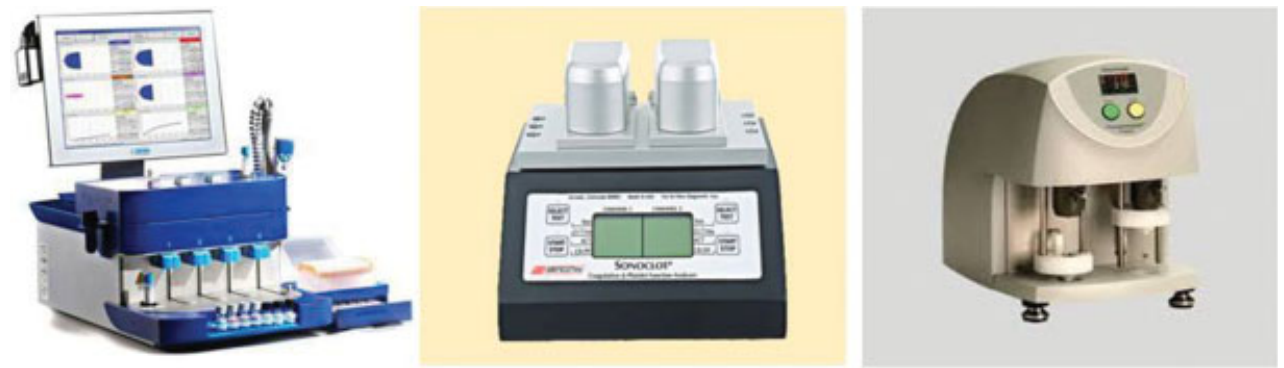

Fig. 1 (A) Platelet aggregometry using rotational thromboelastometry; (B) platelet aggregometry using Sonoclot; (C) platelet aggregometry using thromboelastography.

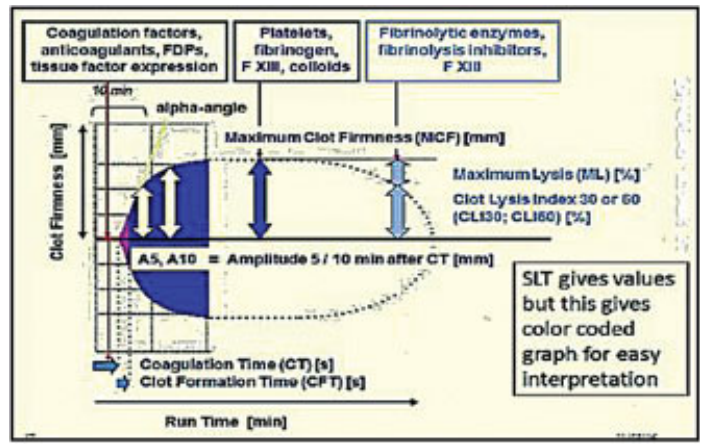

Fig. 2 Color-coded graph for interpretation.

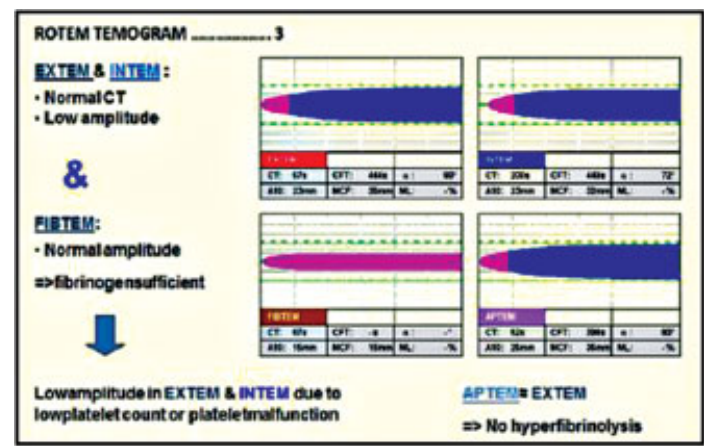

Fig. 3 Rotational thromboelastometry TEMOGRAM with decreased fibrinogen. CT, computed tomography.

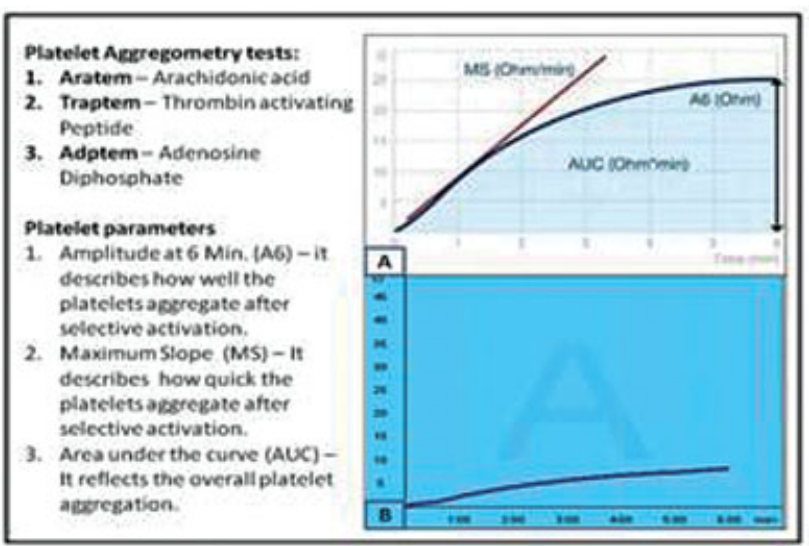

Fig. 4 Rotational thromboelastometry platelet activators and parameters: (A) Normal platelet function test (PFT) and (B) decreased PFT-hyperfibrinolysis.

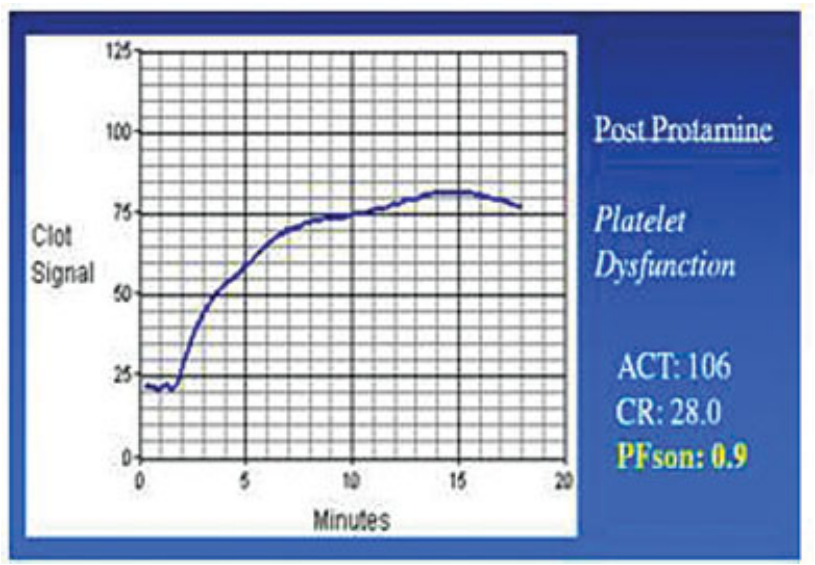

Fig. 5 Sonoclot PFson curve decreased with thrombocytopenia. $A C T$, activated coagulation time; $C R$, clot rate.

\section{Restrictive Blood Transfusion is the Norm in Cardiac ECMO is 2022}

Applying preemptive, restricted diagnostic, and therapeutic strategies of blood transfusion can guide which blood component therapy is to be given. In this era of restrictive transfusion practices, this is a cost-effective strategy of PBM as well as it takes care of health economics of our country; ROTEM platelet function-based testing that is algorithm based and is done in a goal directed transfusion strategy model gives the best guidance to the correct blood product usage of excessive red blood and yellow blood products. If latter is applied in a timely manner, it is costeffective as well. ${ }^{6,7}$

\section{Is There a Role of Factor Concentrates in India?}

Unlike Europe and United States, the four factor concentrates of fibrinogen and prothrombin are poorly marketed in India. Reliance in most cardiac centers today till date is more on the availability of cryoprecipitates, fresh frozen plasma, and platelet concentrates (both random and single-donor platelets) from the blood bank. Giving individual factor concentrates of antithrombin III, factor XI, factor XIII along with Von Willebrand's factor concentrates is still a restraint in most centers. These factor concentrates act faster and should be 
used more often. Though challenging to obtain from the market if latter are given in a timely manner, it would nip the problem of excessive bleeding in the ECMO and cardiac surgical patient in the bud.

\section{Anticoagulation on ECMO Is an Enigma}

Anticoagulation on ECMO is an enigma, but the main emphasis should at all times be on maintaining the hemoglobin and preventing anemia. ${ }^{8}$ In ECMO and cardiac surgery patients, where stakeholders are too many and margin of error is too narrow, preventing anemia and atonicity and being prepared for blood and factor concentrates with right monitoring, of maintaining hemostasis, detecting hypovolemia and coagulopathy are major constraints of a PBM strategy. The three pillars of PBM involve a multimodal, multidisciplinary approach with the aim to resuscitate early, needs preoperative implementation. The Indian scenario of PBM needs awareness, with recommendations and implementation of guidelines. Improving primary healthcare, optimizing anemia, improving blood bank services in tier 2 and 3 cities, and implementing guidelines on the ground in the operating room and intensive care units are to be enacted fast with discipline and teamwork. All this will aid to control bleeding and coagulopathy in the cardiac surgical and ECMO patient and thus prevent a disaster of increased morbidity and mortality!
Conflict of Interest

None declared.

\section{References}

1 Göbel K, Eichler S, Wiendl H, Chavakis T, Kleinschnitz C, Meuth SG. The coagulation factors fibrinogen, thrombin, and factor XII in inflammatory disorders-a systematic review. Front Immunol 2018;9:1731. Doi: 10.3389/fimmu.2018.01731

2 Pagano D, Milojevic M, Meesters MI, et al. 2017 EACTS/EACTA guidelines on patient blood management for adult cardiac surgery. Eur J Cardiothorac Surg 2018;53(01):79-111

3 Gandhi A, Görlinger K, Nair SC, et al. Patient blood management in India - review of current practices and feasibility of applying appropriate standard of care guidelines. A position paper by an interdisciplinary expert group. J Anaesthesiol Clin Pharmacol 2021;37(01):3-13

4 Cartwright B, Bruce HM, Kershaw G, et al. Hemostasis, coagulation and thrombin in venoarterial and venovenous extracorporeal membrane oxygenation: the HECTIC study. Sci Rep 2021;11(01):7975

5 Bhardwaj V, Kapoor PM. Platelet aggregometry interpretation using ROTEM - PART - II. Ann Card Anaesth 2016;19(04):584-586

6 Karanjkar A, Kapoor PM, Sharan S, Bhardwaj V, Malik V. A prospective randomized clinical trial of efficacy of algorithm-based point of care guided hemostatic therapy in cyanotic congenital heart disease surgical patient. J Cardiac Crit Care TSS 2019;03(01):08-16

7 Sharan S, Gandhi A, Kapoor PM. Utility of platelet function tests: a recent review round up. Journal of Cardiac Critical Care TSS 2019; 03(01):24-27

8 Bartlett RH. Physiology of extracorporeal gas exchange. Compr Physiol 2020;10(03):879-891 